\title{
A!
}

This is an electronic reprint of the original article.

This reprint may differ from the original in pagination and typographic detail.

Savin, Alexander; Pekola, Jukka; Flyktman, J.T.; Anthore, Anne

\section{Cold electron Josephson transistor}

Published in:

Applied Physics Letters

DOI:

$10.1063 / 1.1756192$

Published: $24 / 05 / 2004$

Document Version

Publisher's PDF, also known as Version of record

Please cite the original version:

Savin, A., Pekola, J., Flyktman, J. T., \& Anthore, A. (2004). Cold electron Josephson transistor. Applied Physics Letters, 84(21), 4179-4181. https://doi.org/10.1063/1.1756192

This material is protected by copyright and other intellectual property rights, and duplication or sale of all or part of any of the repository collections is not permitted, except that material may be duplicated by you for your research use or educational purposes in electronic or print form. You must obtain permission for any other use. Electronic or print copies may not be offered, whether for sale or otherwise to anyone who is not an authorised user. 


\section{Cold electron Josephson transistor}

A. M. Savin, J. P. Pekola, J. T. Flyktman, and A. AnthoreF. Giazotto

Citation: Appl. Phys. Lett. 84, 4179 (2004); doi: 10.1063/1.1756192

View online: http://dx.doi.org/10.1063/1.1756192

View Table of Contents: http://aip.scitation.org/toc/apl/84/21

Published by the American Institute of Physics

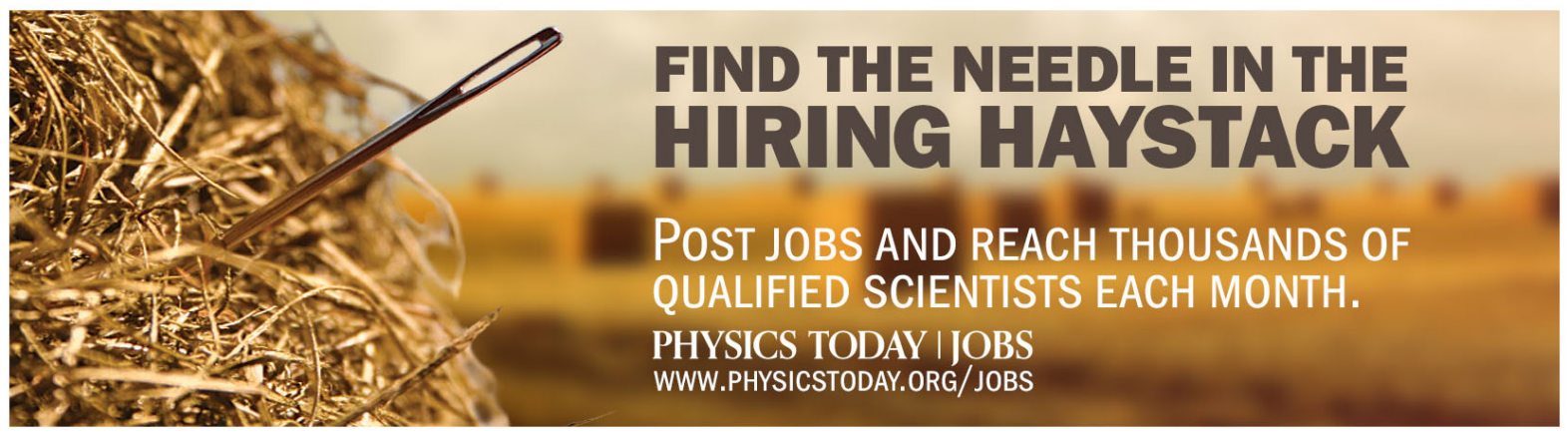




\title{
Cold electron Josephson transistor
}

\author{
A. M. Savin, ${ }^{\text {a) }}$ J. P. Pekola, J. T. Flyktman, and A. Anthore \\ Low Temperature Laboratory, Helsinki University of Technology, P.O. Box 2200, FIN-02015 HUT, Finland \\ F. Giazotto \\ NEST-INFM and Scuola Normale Superiore, I-56126 Pisa, Italy
}

(Received 20 February 2004; accepted 7 April 2004; published online 6 May 2004)

\begin{abstract}
A superconductor-normal metal-superconductor mesoscopic Josephson junction has been realized in which the critical current is tuned through normal current injection using a symmetric electron cooler directly connected to the weak link. Both enhancement of the critical current by more than a factor of two, and supercurrent suppression have been achieved by varying the cooler bias. Furthermore, this transistor-like device demonstrates large current gain $(\sim 20)$ and low power dissipation. () 2004 American Institute of Physics. [DOI: 10.1063/1.1756192]
\end{abstract}

Transport dynamics in mesoscopic structures where normal metals $(\mathrm{N})$ are coupled with superconductors (S) are currently the focus of extensive research. ${ }^{1,2}$ This stems mainly from the relevance these systems have both from the fundamental physics point of view and in light of their possible exploitation in nanoelectronics. In diffusive SNS junctions, where the length of the $N$ region exceeds the elastic mean free path, coherent sequential Andreev scattering ${ }^{3}$ between the superconductors may lead to a continuum spectrum of resonant levels ${ }^{1}$ responsible for carrying the supercurrent flow through the structure. The Josephson current is given by supercurrent spectrum weighted by the occupation number of correlated electron-hole pairs that is determined by the quasiparticle energy distribution in the $N$ region of the junction. By changing the latter through current injection from additional nonsuperconducting terminals connected to the $N$ region $^{4}$ both supercurrent suppression ${ }^{5}$ as well as its sign reversal ( $\pi$-transition) were demonstrated. ${ }^{6}$ As predicted in Refs. 7 and 8, the distinctive quasiparticle distribution existing in the $N$ region of a biased SINIS structure (where $I$ stands for an insulating barrier) is also well suited to control the Josephson coupling in a long SNS weak link, allowing either large supercurrent enhancement or efficient suppression with respect to equilibrium.

In this letter, we present the implementation and characterization of a four-terminal superconducting structure (see Fig. 1) consisting of a SNS mesoscopic junction integrated with a SINIS electron cooler. A similar device was considered but not successfully operated in Ref. 7. In this transistor, the maximum supercurrent flowing in the SNS junction is controlled by voltage biasing the SINIS line whose $N$ region is shared with the Josephson junction. Low temperature transport measurements show enhancement of the critical current under hot quasiparticle extraction by more than a factor of two with respect to equilibrium. In addition this device demonstrates low power dissipation and large current gain.

The sample (shown in Fig. 1) consists of a $\mathrm{Cu}$ island, $0.37 \mu \mathrm{m}$ wide and $30 \mathrm{~nm}$ thick, symmetrically connected at its ends via insulating barriers (with normal-state resistance

${ }^{a)}$ Electronic mail: savin@boojum.hut.fl
$\mathcal{R}_{T} \simeq 240 \Omega$ ) to two 60 -nm-thick $\mathrm{Al}$ reservoirs, thus realizing a SINIS cooler. The Josephson junction instead consists of an $\mathrm{Al} / \mathrm{Cu} / \mathrm{Al}$ SNS weak link (with normal-state resistance $\mathcal{R}_{N}=11.5 \Omega$ ), whose $N$ region is shared with the SINIS line. The minimum interelectrode separation in the SNS junction of the present device is $L_{J} \simeq 0.4 \mu \mathrm{m}$. The structure was fabricated on a thermally oxidized $\mathrm{Si}$ substrate by electron beam lithography and three-angle shadow-mask evaporation. The electrical characterization was performed at different bath temperatures down to $70 \mathrm{mK}$ in a dilution refrigerator. From low-temperature resistance measurements we deduced the Cu diffusion coefficient $D \approx 10 \mathrm{~cm}^{2} / \mathrm{s}$. This low value of $D$ is probably caused by significant intermixing of the materials at the NS interface leading to the strong reduction of the electron mean free path in the weak link. The Al energy gap, $\Delta=169 \mu \mathrm{eV}$, was inferred from the low-temperature current-voltage characteristic of the SINIS line (see Fig. 4). The coherence length $\xi_{N}=\sqrt{\hbar D / \Delta} \approx 62 \mathrm{~nm}$ is then much smaller than $L_{J}$, providing the frame of the long junction regime.

The experiment consists of sweeping the $I_{\mathrm{SNS}}$ current across the SNS junction while measuring its differential re-

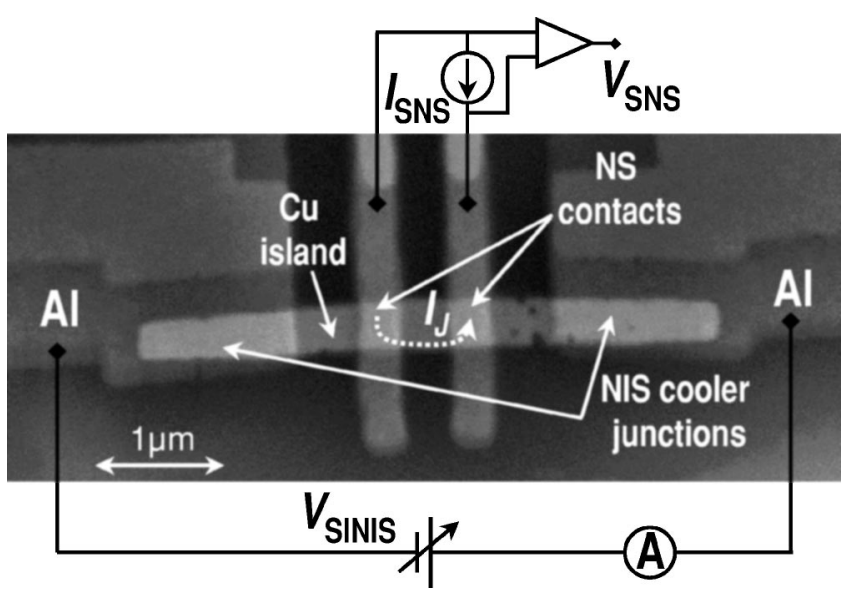

FIG. 1. Scanning electron micrograph of a typical structure including a sketch of the measurement circuit. Two superconducting $\mathrm{Al}$ electrodes are connected through insulating barriers to a $\mathrm{Cu}$ island to realize a symmetric SINIS electron cooler. The supercurrent $I_{J}$ in the $\mathrm{Al} / \mathrm{Cu} / \mathrm{Al}$ junction is tuned upon voltage biasing the SINIS control line. 

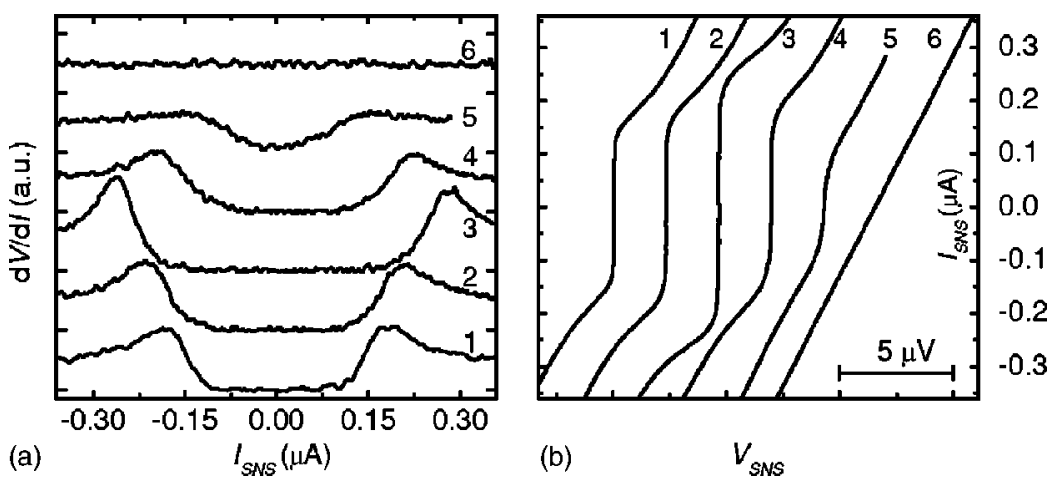

FIG. 2. Selected $d V / d I$ vs $I_{\text {SNS }}$ (a) and current-voltage characteristics (b) of the SNS junction at $T_{\text {bath }}=72 \mathrm{mK}$ for different $V_{\text {SINIS }}$ values (all curves are offset for clarity): 1-0, 2-194 $\mu \mathrm{V}, 3-300 \mu \mathrm{V}, 4-342 \mu \mathrm{V}, 5-355 \mu \mathrm{V}$, $6-938 \mu \mathrm{V}$. Curves in (b) were obtained by numerical integration of the corresponding ones in (a). sistance $d V / d I$ at different values of voltage bias ( $V_{\text {SINIS }}$ ) across the SINIS control line. Figure 2(a) shows a subset of $d V / d I$ vs $I_{\text {SNS }}$ characteristics measured at the bath temperature $T_{\text {bath }}=72 \mathrm{mK}$ for several $V_{\text {SINIS }}$. The curves display a nonhysteretic behavior characteristic for overdamped junctions. ${ }^{9}$ In the case of a SNS weak link the effect of thermal fluctuations on the smearing of the voltage-current characteristic is stronger ${ }^{10}$ than predicted by the model for resistively shunted junction. ${ }^{11}$ We have chosen to define the experimental critical current as the current where the differential resistance reaches $\mathcal{R}_{N} / 2{ }^{12}$ Notably, upon increasing $V_{\text {SINIS }}$, the current range where the differential resistance vanishes widens initially, thus reflecting an enhancement of $I_{J}$, being maximized at a voltage corresponding to $V_{\text {SINIS }}$ $=300 \mu \mathrm{V} \simeq 1.8 \Delta / e^{13}$ [curve labeled as 3 in Fig. 2(a)]; then, further increase of bias leads to a monotonic decay and to a complete suppression of $I_{J}$ at larger voltages [curve labeled as 6 in Fig. 2(a)]. This nonmonotonic behavior is seen in the corresponding $I-V$ curves in Fig. 2(b).

The observed behavior is due to the relation existing between the observable maximum supercurrent $I_{J}$ and the quasiparticle energy distribution in the weak link. In the present experimental situation of large $L_{\text {SINIS }}$, inelastic electron-electron relaxation forces the electron system to retain a local thermal (quasi)equilibrium. As a consequence, the quasiparticle energy distribution can be described with a Fermi-Dirac function at an effective electron temperature $T_{e}$. The temperature $T_{e}$ is determined by the balance between two heat flows:

$$
\mathcal{P}\left(V_{\text {SINIS }}, T_{e}, T_{\text {bath }}\right)+\mathcal{P}_{e-\text { bath }}\left(T_{e}, T_{\text {bath }}\right)=0 .
$$

The first term accounts for the net heat current $\mathcal{P}$ transferred from the $N$ island to the superconductors upon biasing the SINIS line: ${ }^{13}$

$$
\mathcal{P}=\frac{2}{e^{2} \mathcal{R}_{T}} \int_{-\infty}^{\infty} n(E)\left[f_{0}\left(\tilde{E}, T_{e}\right)-f_{0}\left(E, T_{\text {bath }}\right)\right] \tilde{E} d E,
$$

where $\widetilde{E}=E-e V_{\mathrm{SINIS}} / 2, f_{0}(E, T)$ is the Fermi-Dirac distribution function and $n(E)=\left|\operatorname{Re}\left[(E+i \Gamma) / \sqrt{(E+i \Gamma)^{2}-\Delta^{2}}\right]\right|$ is the (smeared by nonzero $\Gamma$ ) BCS density of states of the superconductor. ${ }^{15}$ Equation (2) is symmetric in $V_{\text {SINIS }}$, being maximized slightly below $|2 \Delta / e|$. The second term accounts for energy transfer from electrons to the phonons of the normal island at the temperature $T_{\text {bath }}$ and is equal to $\mathcal{P}_{e}$-bath $=\Sigma \mathcal{V}\left(T_{e}^{5}-T_{\text {bath }}^{5}\right),{ }^{16}$ where $\mathcal{V}$ is the volume of the $N$ island and $\Sigma \approx 2 \mathrm{nWK}^{-5} \mu^{-3}$ for copper. ${ }^{13}$ The temperature $T_{e}$ in the weak link thus strongly depends on $V_{\text {SINIS }}$ and can be smaller than $T_{\text {bath }} \cdot{ }^{17}$ At low temperature (i.e., $k_{B} T_{\text {bath }} \ll \Delta$ ), in a long SNS junction, $I_{J}$ is predicted to decrease exponentially as $T_{e}$ increases $^{18}$ in the regime where $k_{B} T_{e} \gg E_{\mathrm{Th}}$ $=\hbar D / L_{J}^{2}$. Thus, upon biasing the SINIS line, $I_{J}$ will be changed with respect to equilibrium (i.e., at $V_{\text {SINIS }}=0$ ), due to the modification of $T_{e}$ that now differs from $T_{\text {bath }}$.

In Fig. 3(a) we plot the extracted $I_{J}$ values as a function of $V_{\text {SINIS }}$ at three different bath temperatures. For all displayed temperatures, the critical current increases monotonically up to about $V_{\text {SINIS }} \simeq 1.8 \Delta / e$ as expected from the reduction of $T_{e}$ by cooling. Then, further increase of bias voltage leads to an efficient supercurrent suppression due to electron heating. The equilibrium critical current (i.e., at $V_{\text {SINIS }}=0$ ) vs $T_{\text {bath }}$ is displayed in Fig. 3(b). The $I_{J}$ behavior follows a characteristic trend, decreasing upon rising the temperature, but it differs from the temperature dependence predicted by quasiclassical Green-function theory. ${ }^{1}$ The discrepancy can be ascribed to the uncertainty in the determination of the actual values of critical current, relatively narrow temperature range where it was observed and thermal decoupling between electrons and bath at temperatures below $200 \mathrm{mK}$. In Fig. 3, we show the expected critical current dependence on $V_{\text {SINIS }}$ at $T_{\text {bath }}=283 \mathrm{mK}$ obtained from the solution of Eqs. (1) and (2) to determine the effective electron temperature $T_{e}$ upon biasing the SINIS line, and assuming a linear behavior of the critical current $I_{J}$ vs $T_{e}$ below about $350 \mathrm{mK}$, the slope of the linear dependence being inferred from the measured $I_{J}\left(T_{\text {bath }}\right)$. For this calculation, we assumed the already given parameters for the SINIS line and $\Gamma=1.8$ $\times 10^{-3} \Delta$ estimated from the ratio $(\simeq \Gamma / \Delta)$ of the lowtemperature SINIS conductance at low and high bias. ${ }^{14}$ The

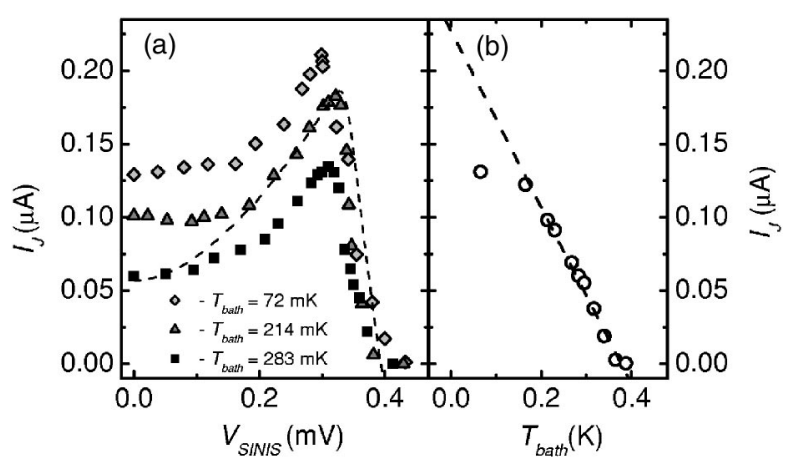

FIG. 3. (a) Critical current $I_{J}$ vs control voltage $V_{\text {SINIS }}$ at three different bath temperatures; (b) equilibrium supercurrent $\left(V_{\text {SINIS }}=0\right)$ vs bath temperature. Dashed line in (a) represents curve obtained from energy balance Eq. (1) and the linear approximation of $I_{J}\left(T_{\text {bath }}\right)$ shown in (b). 


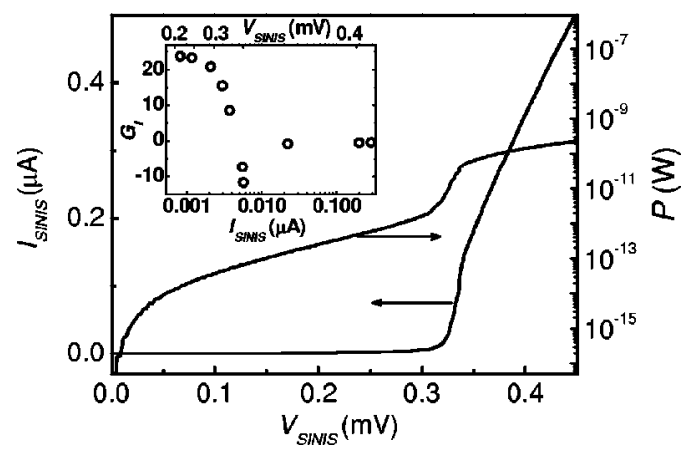

FIG. 4. Current-voltage characteristic (left axis) and power dissipation $P$ $=V_{\text {SINIS }} I_{\text {SINIS }}$ (right axis) of the SINIS line at $T_{\text {bath }}=72 \mathrm{mK}$. The inset shows the measured differential current gain $\mathbf{G}_{I}$ against $I_{\mathrm{SINIS}}$ at the same temperature (also displayed is the dependence on $V_{\text {SINIS }}$ ).

resemblance between calculation and experiment is evident although details of the former one are dictated by the $I_{J}$ dependence on temperature, which we cannot extrapolate reliably. To better characterize our device, we show in Fig. 4 (right axis) the dissipated power $P$ against $V_{\text {SINIS }}$ in the SINIS line at $T_{\text {bath }}=72 \mathrm{mK}$. The plot reveals that in the bias voltage region of significant critical current enhancement (i.e., in the $200-300 \mu \mathrm{V}$ bias range) $P$ obtains values of the order of $10^{-13} \mathrm{~W}$, while in the regime of supercurrent suppression (i.e., for $V_{\text {SINIS }}>300 \mu \mathrm{V}$ ) some tens of $\mathrm{pW}$. This demonstrates the low power dissipation intrinsic to the structure. ${ }^{8}$ The $P$ behavior is directly related to the normal current flow in the control line. The latter is displayed on the left-hand side axis of Fig. 4 and shows that control currents as low as a few nA are necessary to enhance the critical current, while of about $100 \mathrm{nA}$ to suppress it. The differential current gain $\mathbf{G}_{I}=d I_{J} / d I_{\text {SINIS }}$ against $I_{\text {SINIS }}$ is shown in the inset of Fig. 4. Notably, $\mathbf{G}_{I}$ obtains values exceeding 20 in the hot quasiparticle extraction regime, while of about -11 in the voltage region of supercurrent suppression. We note that higher $\mathbf{G}_{I}$ values, as well as lower power dissipation and control currents, could be attained by optimizing the structure design. ${ }^{8,14}$

In summary, we have demonstrated experimentally control of Josephson coupling under hot quasiparticle extraction in a four-terminal superconducting structure. Our experimen- tal result shows the potential of a SINIS line as a basis of a promising class of mesoscopic transistors with high current gain.

The authors acknowledge T. T. Heikkilä, F. Carillo, R. Fazio, P. J. Hakonen, F. W. J. Hekking, and F. Taddei for discussions, and Academy of Finland for financial support (TULE Program). One of the authors (F.G.) would like to acknowledge the Large Scale Installation Program ULTI-3 of the European Union for the kind hospitality and for financial support.

${ }^{1}$ W. Belzig, F. K. Wilhelm, C. Bruder, G. Schön, and A. D. Zaikin, Superlattices Microstruct. 25, 1251 (1999).

${ }^{2}$ C. J. Lambert and R. Raimondi, J. Phys.: Condens. Matter 10, 901 (1998).

${ }^{3}$ A. F. Andreev, Zh. Eksp. Teor. Fiz. 46, 1823 (1964).

${ }^{4}$ F. K. Wilhelm, G. Schön, and A. D. Zaikin, Phys. Rev. Lett. 81, 1682 (1998); A. F. Volkov, ibid. 74, 4730 (1995); S.-K. Yip, Phys. Rev. B 58, 5803 (1998).

${ }^{5}$ A. F. Morpurgo, T. M. Klapwijk, and B. J. van Wees, Appl. Phys. Lett. 72, 966 (1998).

${ }^{6}$ J. J. A. Baselmans, A. F. Morpurgo, B. J. van Wees, and T. M. Klapwijk, Nature (London) 397, 43 (1999); R. Shaikhaidarov, A. F. Volkov, H. Takayanagi, V. T. Petrashov, and P. Delsing, Phys. Rev. B 62, R14649 (2000); J. Huang, F. Pierre, T. T. Heikkilä, F. K. Wilhelm, and N. O. Birge, ibid. 66, 020507 (2002).

${ }^{7}$ J. J. A. Baselmans, Ph.D. thesis, University of Groningen, The Netherlands, 2002.

${ }^{8}$ F. Giazotto, F. Taddei, T. T. Heikkilä, R. Fazio, and F. Beltram, Appl. Phys. Lett. 83, 2877 (2003).

${ }^{9}$ K. K. Likharev, Rev. Mod. Phys. 51, 101 (1979).

${ }^{10}$ T. Hoss, C. Strunk, T. Nussbaumer, R. Huber, U. Staufer, and C. Schönenberger, Phys. Rev. B 62, 4079 (2000).

${ }^{11}$ V. Ambegaokar and B. I. Halperin, Phys. Rev. Lett. 22, 1364 (1969).

${ }^{12}$ P. Dubos, H. Courtois, B. Pannetier, F. K. Wilhelm, A. D. Zaikin, and G. Schön, Phys. Rev. B 63, 064502 (2001).

${ }^{13}$ M. M. Leivo, J. P. Pekola, and D. V. Averin, Appl. Phys. Lett. 68, 1996 (1996).

${ }^{14}$ J. P. Pekola, T. T. Heikkilä, A. M. Savin, J. T. Flyktman, F. Giazotto, and F. W. J. Hekking, Phys. Rev. Lett. 92, 056804 (2004).

${ }^{15}$ The parameter $\Gamma$ allows quasiparticle states within the energy gap $\Delta$ (Ref. 14).

${ }^{16}$ F. C. Wellstood, C. Urbina, and J. Clarke, Phys. Rev. B 49, 5942 (1994).

${ }^{17}$ M. L. Roukes, M. R. Freeman, R. S. Germain, and R. C. Richardson, Phys. Rev. Lett. 55, 422 (1985).

${ }^{18}$ A. D. Zaikin and G. F. Zharkov, Sov. J. Low Temp. Phys. 7, 184 (1981); F. K. Wilhelm, A. D. Zaikin, and G. Schön, J. Low Temp. Phys. 106, 305 (1997). 